\title{
A Branching Process for Convergent Product Optimization
}

\author{
Ermia Aghasi ${ }^{1 *}$, Mansour Momeni ${ }^{1} \&$ Mohammad Ali Shah Hoseini ${ }^{1}$ \\ ${ }^{1}$ Faculty of Management, University of Tehran, Tehran, Iran \\ *Ermia Aghasi, E-mail: ermia.aghasi@gmail.com
}

Received: June 16, 2017

Accepted: July 8, 2017

Online Published: July 13, 2017

doi:10.22158/rem.v2n4p1

URL: http://dx.doi.org/10.22158/rem.v2n4p1

\begin{abstract}
We consider a discrete time branching process where the population consists of $k$ types of convergent products, an action is chosen for that which affects the lifetime, the number and types of its functions, and the profit received. The problem of maximizing the expected profit is shown to be equivalent to a generalized Markov decision problem of maximizing the expected profit is shown to be equivalent to a generalized Markov decision problem where the $(k \times k)$ transition matrices are non-negative but not necessarily sub stochastic.
\end{abstract}

\section{Keywords}

convergent product, branching process

\section{Introduction}

Research over the past 30 years has shown that the NPD process is based on a series of development stages that are interpolated by a series of evaluative stages. These evaluation stages can be better understood as "gates" (Cooper, 1990) or "convergent points" (Hart \& Baker, 1994) in that they can navigate managers to avoid go and no-go errors during the development process. Within each evaluation gate, management uses pre-specified criteria to assess whether different tasks have been performed efficiently and effectively. These criteria act as "guide posts" against which the performance of the NPD effort can be evaluated and adjustments made, if necessary. To the extent that these guideposts are derived from the corporate and new product strategy of the firm and are focused to the specific requirements of each stage of the NPD process, they can help reduce managerial uncertainty and identify areas where additional attention and resources are needed. Furthermore, they can inform the strategic decision making process of the firm as they can assist management to plan what competencies and resources to develop and deploy throughout the NPD effort.

A New Product Development (NPD) project is not just a series of predictable steps that can be identified and planned in advance (Schilling \& Hill, 1998). For many product development projects, particularly those with some non-trivial level of technology uncertainty, the resulting capabilities of the product and the exact means to achieve the product are not known with certainty at the start of the 
development project. Often, before project execution starts, there is no precise understanding of the detailed project tasks, task sequence, task interdependencies and task times (Tatikonda \& Rosenthal, 2000).

According to Lettice et al. (2006), performance assessment of NPD projects is a central issue in innovation management research. There have been controversial discussions in the literature about success measures (e.g., Ernst, 2001; Pinto \& Slevin, 1988). The primary challenge of a NPD project is to achieve all of the project goals and objectives while adhering to project constraints (Teimoury et al., 2011). Although in general terms project performance is recognized as a multidimensional parameter (de Oliveira et al., 2012) the most cited project output variables are comprised of cost, schedule, technical performance outputs, and customer satisfaction. Some authors (Dvir \& Lechler, 2004; Ernst, 2001; Pinto \& Slevin, 1988; Verworn et al., 2008) determined the efficiency of the NPD project, by assessing the degree of agreement between financial and personnel resources planned during the fuzzy front end and those actually required, and the accordance with the achievement of milestones. Other authors measured the overall satisfaction of project managers with the new product development process and results. This measurement is based on a factor introduced by Lechler (1997).

Tatikonda and Rosenthal (2000) analysed a cross-sectional survey sample of 120 completed new product development projects from a variety of assembled products industries and used, as a project execution success measure, a weighted sum of the degree of achievement of each of the three central project objectives technical performance, product unit-cost, and time-to-market, where the weights are based on the relative importance of each objective for the given project.

Verworn (2009) used a four-item scale for measuring NPD performance. The author asked informants to assess the degree of a new product's success relative to project goals on sales, profit margin, return on assets and return on investment.

Most of the project performance evaluation methods used by many organizations do not explicitly consider key input variables that add value for the client (Farris et al., 2006). This study attempts to bridge this gap using two performance indexes usually used in "Lean Production" sector: "waste time" and "type of wastes".

Several researchers have suggested a contingency approach to new product development (Balachandra \& Friar, 1997; Drejer, 2002; Khurana \& Rosenthal, 1998). Tatikonda and Rosenthal (2000) defined a conceptual framework that addresses three project execution methods: formality, project management autonomy and resource flexibility that project managers may employ, either singly or collectively, to achieve greater project execution effectiveness in new product development projects. March-Chorda et al. (2002) identified three major critical factors for the success of product development and innovation: (i) top management support, (ii) product development planning, and (iii) analysis of market requirements. Liker and Morgan (2006) conducted an in-depth study of Toyota's approach to process and product development and they identified 13 management principles considered to be the basis of the Lean product development. 
These principles are defined in three frameworks: process, people, and tools and technology. Songa and Noh (2006) investigated critical factors affecting the likelihood of new product success and effective New Product Development (NPD) models for Korean high-tech firms. Empirical results suggest that successful projects differ from unsuccessful projects in project environment, skills and resources, project leadership, strategic fit, efficient NPD process, and effective product-positioning strategies.

This paper presents results for and potential applications of the optimal control of branching processes. Despite large literatures on both branching processes and dynamic programming, the control of branching processes is a relatively new concept. Sevast'yanov and zubkov (1974) and mandl (1967) have studied certain aspects of this problem but their viewpoints are different from those presented here.

One appeal of branching processes is that they are easy to analyze. As a result of the assumption that the individuals act independently of each other. For example, an ordinary branching process with $\mathrm{k}$ types of products can always be formulated as an infinite state Markov decision chains, by applying some branching process ideas we can reduce the problem to a finite state decision problem and obtain a corresponding simplification of the control theoretic results and computational procedures.

This paper is primarily concerned with the problem of controlling a discrete time branching process where the population consists of $\mathrm{k}$, a finite number, types of convergent products. After formulating this model, the finite planning horizon case is treated. There we show that some policy of control of a simple form is optimal over a much wider class of policies, and we show how to compute such an optimal policy by solving a second Markov decision problem whose $(k \times k)$ transition matrices are nonnegative but not necessarily sub stochastic.

In this paper, we propose a methodology for convergent product. The remainder of our work is organized as follows. Next, we develop the problem. In Section 3 the proposed branching process is given through mathematical formulations. In Section 4 a case study is conducted to show the implementation of the methodology. We conclude in Section 5.

\section{Proposed Problem}

Convergent Product (CP) is a specific process in New Product Development (NPD) in which Product Design (PD) is a significant part. The aim of $\mathrm{CP}$ is to acquire indices or functions to be collected through Customer Relationship Management (CRM) and then should be purified and updated. Traditionally, Quality Function Deployment (QFD) is performed instead of CP. But the limitation of QFD is mainly its focus on quality merely while a group of other indices can be effective such as customers' views of Balanced Score Card (BSC) indices. Thus, in this research a CP based process is proposed to overcome common drawbacks of past methods and provide integrated decision making mechanism for top management.

To do that a two stage process is designed as follows:

1) An environment is proposed to collect customers' views and integrate it with purification and 
product functions update. The aim here is to identify the indices and present products with alternative function to satisfy customers' utility.

2) Evaluation of functions and indices of products, which was performed using statistical or data mining techniques in the previous literature, is aimed here to include uncertainty of customers' views and will be modelled by a branching process.

Acquiring customers' views on product functions is performed using internet based questionnaire to assess customer satisfaction measure. Then, using a branching process the significant functions with respect to attributes are obtained and the expected profit is computed. The objective in this proposed tree is finding a set of functions and sub-functions that optimize the objectives of the problem such as maximizing the expected profit. This way and regarding the uncertainty of the problem a modified branching process is designed to solve the problem. The aim here is to develop a modified branching process to obtain a set of functions and sub-functions with respect to the objectives of the problem.

\section{Mathematical Formulations}

We consider a branching process of $\lambda$ type, denoted $\mathrm{i}=1, \ldots, \mathrm{k}$. The process is observed at successive times or epochs. Denoted $n=0,1, \ldots$. At any such time the process is observed to be in a state $\mathrm{s}=\left(\mathrm{s}_{1}, \ldots, \mathrm{s}_{\mathrm{k}}\right)$. Where the nonnegative integer $\mathrm{s}_{\mathrm{i}}$ denotes the number of individuals of type $\mathrm{S}$ thus consists of all k- product vectors whose products are nonnegative integers, and the state at each time describes the composition of the corresponding generation or population of all product types produced.

Each time a type/individual is observed a corresponding action $a$ is selected from a prescribed finite set $A$ the actions selected are thus allowed to vary from individual of the same type as well as from generation to generation.

Each time an action $a$ is selected for a type $I$ individual, the profit $\mathrm{r}_{\mathrm{i}}(\mathrm{a})$ is received (assume $\infty<r,(a)<\infty)$. The generation profit received at a particular time is the sum of the individual profits over the population of individuals. The contribution to the generation profit at a particular time by a particular individual thus does not depend on the composition of and actions selected for the other individuals in the population.

The action selected for an individual affects the number of functions that individual has in the next generation. For each $k=$ dimensional vector $t=\left(t_{1}, \ldots, t_{k}\right)$ of nonnegative integers, let $p_{1}(t, a)$ be the probability that a particular product of type $t$, whose corresponding action is $a$, will produce exactly $t_{1}$ type functions , ..., and $t_{\lambda}$ type $k$ functions (we assume $\sum_{i p i}(t, a)=1$ ). Note that, analogous to the ordinary multi type branching process situation, each product lives exactly one period and the set of functions produced by a particular product is independent of the other products in the same generation and of their actions.

Corresponding to each $\mathrm{p}_{\mathrm{i}}(0, \mathrm{a})$ is the row vector $\mathrm{m}_{\mathrm{i}}(\mathrm{a})=\left(\mathrm{m}_{\mathrm{a}}(\mathrm{a}), \ldots \mathrm{m}_{\mathrm{ik}}(\mathrm{a})\right)$, where $\mathrm{m}_{\mathrm{ij}}(\mathrm{a})$ equals the expected number of type $j$ functions produced by a single type $i$ product under action $a$. Throughout all that follows, we make the following assumption: 


$$
m_{i j}(a)<\infty \text { for all } a \in A_{i} \text { and } i, j=1, \ldots, k
$$

A decision rule is a function $f$ on the state space which defines the action to be selected for each product at a particular time. In general, different actions may be selected for different products of the same type, and the selection for a product may depend upon the composition of the population. Let $F$ denote the set of all such decision rules.

A decision rule $f \in F$ is said to be static if the same action is chosen for all products of the same type and this selection is independent of the state $s \in S$. In other words, a static decision rule $\mathrm{f}$ can be defined by a suitable k- dimensional vector $\left(a_{1}, \ldots, a_{k}\right)$ where $\mathrm{a}_{\mathrm{i}}$ indicates the action selected for the type $i$ products. Let $G=A_{1} \times \ldots \times A_{k} \subset F$ denote the set of all static decision rules.

A policy $\pi$ is a sequence $\left(f_{n}\right), n=0,1, \ldots$ of decision rules that correspond to the decision epochs. A policy $\pi$ is said to be stationary if $f_{0}=f_{1}=\ldots, A$ policy is said to be static it $f_{n}$ is a static decision rule for each $\mathrm{n} \geqslant 0$. Let $F^{a}$ and $G^{a}$ denote the sets of all policies and all static policies respectively. Notice that only deterministic, memory less policies are being considered.

Each policy $\pi \in f^{a}$ gives rise to a no stationary Markov chain $(\mathrm{Z}(\mathrm{n}): \mathrm{n}=0,1, \ldots)$ with state space $\mathrm{S}$ and one-step transition probabilities that can be determined from the generating functions of the functions probabilities. Naturally, $\mathrm{Z}(\mathrm{n})$ is the state of the population at epoch $n$. throughout all that follows, regard the state (either $Z(n)$ or $s$ ) as a row vector.

For future purposes, let $\mathrm{R}(\mathrm{s}, \mathrm{f})$ be the total profit received at a particular epoch when the state then is $s$ and the decision rule $f$ is used. Upon knowing the transition probabilities, the expected profit at each epoch under a policy may be computed in a straightforward manner.

\subsection{The Finite Horizon Problem}

Roughly speaking, in the case of a finite horizon $N$, we want to choose decision rules over the periods $n=0,1, \ldots, N-1$ so as to maximize the conditional expectation of the sum of the generation profits over periods $n=0,1, \ldots, N-1$ plus the terminal profit at time $n=N$. The terminal profit is specified by a prescribed column vector $T$ with the interpretation that the terminal profit at time $n-N$. the terminal profit is specified by a prescribed column vector $T$ with the interpretation that the terminal profit received at time $n$ is (the scalar) $s T$ in the event that $\mathrm{Z}(\mathrm{n})=\mathrm{s}$.

Let the positive number a be a discount factor. The N-period discounted profit under policy $\pi=\left\{f_{n}\right\} \in F^{\infty}$ is defined by the random variable

$$
W_{a}^{N}(\pi)=R\left(Z(0) \cdot f_{0}\right)+\alpha R\left(Z(1), f_{1}\right)+\ldots+\alpha^{N-1} R\left(Z(N-1), f_{N-1}\right)+\alpha^{N} Z(N) T
$$

The expected N-period discounted profit under policy $\pi$ is defined by

$$
V_{a}^{N}(s, \pi)=E\left[W_{a}^{N}(\pi) \mid Z(0)=s\right]
$$


If $a=1$, then we have the usual undiscounted case. Notice that $V_{a}^{N}(s, \pi)$ is well defined and finite whenever $N<\infty$ because of equation (1) and the finiteness of each A.

$\mathrm{N}$-period discounted profit defined by

$$
V_{a}^{N}(s)=\sup _{\pi \in F^{\infty}} V_{a}^{N}(s, \pi)
$$

Note that supere mum is over the set of all nonstatic as well as static policies. We also seek an optimal policy, that is, some policy $\pi \in F^{\infty}$ such that

$$
V_{a}^{N}(\pi, s)=V_{a}^{N}(s), \text { all } s \in S .
$$

In this section, we will show that there always exists an optimal policy which is static and, along with the maximum expected profit, can be computed with the usual iterative methods of dynamic programming.

For each static decision rule $f=\left(a_{1}, \ldots, \quad a_{k}\right) \in G$, let $\mathrm{r}(\mathrm{t})$ denote the column vector whose/th product is $r_{1}\left(a_{1}\right)$. Similarly, let M(f) denote the $k \times k$ matrix whose ijth element is $m_{i j}\left(a_{1}\right)$. We now define the optimal return operator ${ }^{\mu}$ a function on k-dimensional Euclidean space $E^{k}$ into itself, by

$$
\mathfrak{R} X=\max _{f \in G}(r(f)+\alpha M(f) X)
$$

Let $\mathfrak{R}$ denote the n-fold composition $\mathfrak{R}$, let $\mathfrak{R}^{0}$ denote the identity operator, and regard $\mathfrak{R}_{\mathrm{X}}$ as a column vector whenever $\mathrm{X}$ is. This leads to the main result of this section.

THEOREM 1. The maximum expected N-period discounted profit satisfies

$$
V_{a}^{N}(s)=s \mathfrak{R}^{N} 7
$$

A static policy which is obtained by setting $f_{n} \in G$ such that

$$
r\left(f_{n}\right)+a M\left(f_{n}\right) \mathfrak{R}^{N-n-1} T=\mathfrak{R}^{N-n} T, \quad n=0,1, \ldots, N-1,
$$

is optimal.

PROOF. First note that if $\pi=\left(f_{n}\right)$ is a static policy, then

$$
V_{a}^{N}(s, \pi)=\operatorname{sr}\left(f_{0}\right)+\alpha s M\left(f_{0}\right) r\left(f_{1}\right)+\ldots+\alpha^{N} s M\left(f_{0}\right) \ldots M\left(f_{N-1}\right) 7:
$$


This is because the ijth element of $M\left(f_{N}\right)$ equals the expected value under $\pi$ of the number of the number of type $\mathrm{j}$ individuals in the $\mathrm{N}$ th generation given the initial generation consists of a single type/product. Hence the static policy $\hat{\pi}$ obtained by (8) satisfies $V_{\alpha}^{N}(s, \pi)=s \Re^{N} \quad T$, so it suffices to show $s \Re^{N} T \geq V^{N}(s, \pi)$ for all $n \in F^{\infty}$ by induction on $\mathrm{N}$.

For $\mathrm{N}=1$, let $\quad \mathrm{S} \in \mathrm{S}$ and $f_{0} \in F \quad$ be arbitrary, and let $a_{i j}$ denote the resulting action selected for the jth type i product. Let $\left(a_{1}, \ldots, a_{k}\right) \in G$ be a static decision rule that satisfies (8) with $\mathrm{N}=1$ and $\mathrm{n}=0$. Then

$$
\left.V_{0}^{i}(s, \pi)=\sum_{i=1}^{k} \sum_{j=1}^{s i}\left(r_{i}\left(a_{i j}\right)+\alpha m_{i}\left(a_{i j}\right) T\right) \leq \sum_{i=1}^{k} \sum_{j=1}^{s_{1}}\left(r_{1}\left(a_{1}\right)+a m_{1}\left(a_{1}\right) T\right)+a m_{1}\left(a_{1}\right) T\right)=s \Re T
$$

where the sum $\sum_{j}^{s_{i}}=0$ whenever $s_{i}=0$, so the result is true for $\mathrm{N}=1$.

Now suppose the theorem is true for $\mathrm{N}=\mathrm{K}$. Let $s \in S$ and $n=\left(f_{n}\right) \in F^{\infty}$ be arbitrary. Denote $\hat{\pi}=\left(f_{1}, f_{2}, \ldots\right)$, so that $\pi=\left(f_{0} \hat{\pi}\right)$. Let $a_{i j}$ denote the action selected for the jth type i product corresponding to $f_{0}$ and s. The

$$
\begin{gathered}
V_{a}^{k+1}(s, \pi)=E\left\{E\left[W_{a}^{k+1}(\pi) \mid Z(1)\right] \mid Z(0)=s\right\} \\
=\sum_{i=1}^{m} \sum_{j=1}^{s_{i}} r_{i}\left(a_{i j}\right)+E\left\{E\left[\alpha W_{a}^{N}(\tilde{\pi}) \mid Z(l)\right] \mid Z(0)=s\right\} \\
=\sum_{i=1}^{m} \sum_{j=1}^{s_{i}} r_{i}\left(a_{i j}\right)+\alpha E\left\{V_{a}^{N}(Z(l), \tilde{\pi}) \mid Z(0)=s\right\} \\
\leq \sum_{i=1}^{m} \sum_{j=1}^{s_{i}} r_{i}\left(a_{i j}\right)+\alpha E\left\{Z(l) \Re^{k} T \mid Z(0)=s\right\} \\
=\sum_{i=1}^{m} \sum_{j=1}^{s_{i}}\left[r_{i}\left(a_{i j}\right)+\alpha m_{i}\left(a_{i j}\right) \Re^{k} T\right]=s \Re^{k+1} T,
\end{gathered}
$$

Theorem 1 says that a branching process decision problem can be solved by solving a related k-state Markov decision chain. To see this, note that (8) requires the recursive computation of the vectors $\mathfrak{R} T, \mathfrak{R}^{2} T$, and so forth. But looking at the definition of $\mathfrak{R}$. It is clear that $\mathfrak{R} T$ and is successors are obtained by solving the k-state Markov decision problem with transition matrices $\mathrm{M}(\mathrm{f})$ and profit vectors $\mathrm{r}(\mathrm{f})$. Of course, the nonnegative transition matrices are not necessarily sub stochastic, but this presents no problem as far as the computations are concerned. Static policies in the original problem correspond to deterministic and memory less polices in the associated problem, and an optimal $\mathrm{N}$-period policy for the associated problem is also optimal (among no static as well as static policies) 
for the original problem. The importance of theorem 1, therefore, is that it provides the correct procedure for calculating optimal polices; the somewhat intuitive result that a static policy is optimal among no static policies is of secondary importance.

\section{Case Study}

This section enumerates an application of controlled branching processes. We have chosen to omit several other management science applications, because the appropriate models do not permit multiple births and thus do not have the computational savings of controlled branching processes.

Now let's consider an example for the convergent product. New products that arrive in a random fashion can be modeled by adding a dummy type, say type 0 . For example, consider a discrete time Process with transition matrices $M(f)$. and suppose we want to add a products with a function, $Y(n)$. Where the k-dimensional vector $Y(n)$ represents the population of functions that are suggested for a product at period $n$ and $\{Y(n)\}$ is a sequence independent and identically distributed random variables (one could even control this function and receive different such controls). We do this by adding to each $M(f)$ a zeroth column of zeros and a zeroth row with the 00th element equal to one and the 0 th element equal to the expected value of the jthfunction of product of $Y(1)$. That is, the expected number of type $\mathrm{j}$ functions to be assembled each period. Then by starting the new process with exactly one type 0 product, there will be exactly one type 0 product each succeeding period, and the process observed at type 1 through $\mathrm{k}$ will be the one we are interested in. Notice that the spectral radius of the new transition matrix equals the maximum of one and the spectral radius of the original transition matrix $M(f)$. Although multiple functions are always present in the convergent product process, if they are excluded from the original process. Then an optimal control and the maximum expected profit can still alternatively be computed by considering the superposition of k-state Markov decision chains, so no computational savings are achieved.

Consider a marketing model, where state $i$ means a customer last chosen function $i$. Here it is useful to allow the size of the population to fluctuate with an convergent product due to external factors such as advertising as well as with promotions and discounts.

Now, consider a case study in an online shopping company. Two digital product groups of DVD players and smart phones are considered. These products can be considered as convergent product by adding or removing functions. The attributes to evaluate the functions are,

1) Price,

2) Product quality,

3) On time delivery,

4) Service quality,

5) Brand,

6) and the functions are,

7) Resolution, 

8) Bluetooth,
9) Internal memory,
10) Monitor size,
11) Weights,
12) Energy consumption.

The sample understudy is 150 customers' views (which their mean values are employed) and the decision matrix is completed by the experts in sale and customer relationship management departments. The costs of adding functions with respect to the attributes and the profit obtained by each product type are collected. Then, using equations (6-11) we can compute an optimal policy for the two products as follows:

- If the functions, "Monitor size" and "Energy consumption" are assemble on the DVD player and according to the attributes, "Price" and "Service quality" the expected profit in 10 periods of selling is obtained to be $78365 \$$.

- If the functions, "Weights", "Resolution" and "Internal Memory" are assemble on the smart phones and according to the attributes, "Bran" and "Product quality" the expected profit in 10 periods of selling is obtained to be 103875 \$.

Thus, with respect to the attributes and the views of the customers the importance weights of the functions show that weights and resolution ranks first and then energy consumption and monitor size are in the second place.

\section{Conclusions}

In this work a new integration between customer views, product functions and economic profit of the producer or supplier was proposed. The decision making problem is of convergent product. First the decision matrix including assembly costs and selling price was formed. Then, by a branching process the weights of the functions and attributes were aggregated. Finally, the expected profit of handling the customers' suggestions is obtained through a Markovian process. The applicability and the validity of the proposed method were tested in a case study.

\section{References}

Fahimeh, R., \& Jie, L. (2014). An intelligent group decision-support system and its application for project performance evaluation. Journal of Enterprise Information Management, 27(3), 278-291. https://doi.org/10.1108/JEIM-12-2013-0096

Filippo, E. C., Maurizio, B., \& Giovanni, M. (2015). Performance analysis of new product development projects: An approach based on value stream mapping. International Journal of Productivity and Performance Management, 65(2), 177-206.

Gill, T. (2008). Convergent products: What functionalities add more value to the base? Journal of Marketing, 72(2), 46-62. https://doi.org/10.1509/jmkg.72.2.46 
Gill, T., \& Lei, J. (2009). Convergence in the high-technology consumer markets: Notall brands gain equally from adding new functionalities to products. Marketing Letters, 20(1), 91-103. https://doi.org/10.1007/s11002-008-9050-5

Goumas, M., \& Lygerou, V. (2000). An extension of the PROMETHEE method for decision making in fuzzy environment: Ranking of alternative energy exploitation projects. Eur. J. Oper. Res, 123, 606-613. https://doi.org/10.1016/S0377-2217(99)00093-4

Greenstein, S., \& Khanna, T. (1997). What does industry convergence mean? In D. B. Yoffie (Ed.), Competing in the age of digital convergence (pp. 201-226). Boston: Harvard Business School Press.

Kaluza, B., Blecker, T., \& Bischof, C. (1999). Implications of digital convergence on strategic management. In S. B. Dahiya (Ed.), The current state of economic science (pp. 2223-2249). Rohtak: Spellbound Publications.

Macharis, C., Springael, J., de Brucker, K., \& Verbeke, A. (2004). PROMETHEE and AHP: The design of operational synergies in multicriteria analysis. Stengthening PROMETHEE with ideas of AHP. Eur. J. Oper. Res, 153(2), 307-317. https://doi.org/10.1016/s0377-2217(03)00153-x

MANDI, P. (1967). An Iterative Method for Maximizing the Characteristic Root of Positive Matrices. Rec, Roum. Math Pures Et Appl. Tome XII, 1312-1322.

Marcin, R., \& Krzysztof, B. (2015). ESTIMATING NEW PRODUCT SUCCESS WITH THE USE OF INTELLIGENT SYSTEMS. Foundations of Management, 6(2).

Ozgen, A., Tuzkaya, G., Tuzkaya, U. R., \& Ozgen, D. (2011). A Multi-Criteria Decision Making Approach for Machine Tool Selection Problem in a Fuzzy Environment. Int. J. Comput. Intell. Syst, 4(4), 431-445. https://doi.org/10.1080/18756891.2011.9727802

Rust, R. T., Thompson, D. V., \& Hamilton, R. W. (2006). Defeating feature fatigue. Harvard Business Review, 84, 98-109.

Sevast'yanov, B. A., \& Zubkov, A. M. (1974). Controlled Branching Processes. Th. Prob. And its Appl., XIX(1974), 14-24. https://doi.org/10.1137/1119002

Tuzkaya, G., Gülsün, B., Kahraman, C., \& Özgen, D. (2010). An integrated fuzzy multi-criteria decision making methodology for material handling equipment selection problem and an application. Expert Syst. Appl, 37(4), 2853-2863. https://doi.org/10.1016/j.eswa.2009.09.004

Ulengin, F., Topçu, Y., \& Sahin, S. O. (2001). An Integrated decision aid system for Bosporous water crossing problem. Eur. J. Oper. Res, 134, 179-192. https://doi.org/10.1016/S0377-2217(00)00247-2

Verworn, B., Herstatt, C., \& Nagahira, A. (2008). The fuzzy front end of Japanese new product development projects: Impact on success and differences between incremental and radical projects. R\&D Management, 38(1), 1-19. https://doi.org/10.1111/j.1467-9310.2007.00492.x 